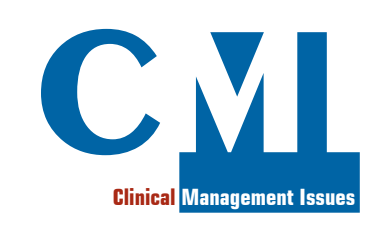

\title{
Acknowledgement to Reviewers (January 2019 - December 2019)
}

The editorial staff of Clinical Management Issues (CMI) would like to thank all the reviewers who, with their support and their active cooperation, have contributed to improving the scientific rigor, precision, and accuracy of the contents.

- Sakir Ahmed

- Osaree Akaraborworn

- Eduardo Anguita

- Shane Belvedere

- Antonio Bertoletti

- Steve Carlan

- Elio Castagnola

- Luis José Catoggio

- Stefania Chetcuti Zammit

- Francesco Cortese

- Adolfo Rafael de Roodt

- Michael Eddleston

- Antonino Faillaci

- Khursheed N. Jeejeebhoy

- Jagadeesh Kalavakunta

- Ramesh Kumaresan
- Marco Lenzi

- Antonella Lezo

- Filomena Longo

- Guglielmo Mantica

- Giuseppe Murdaca

- Dimitri Peterlana

- Anja Potthoff

- Ittichai Sakarunchai

- Vishal Sharma

- Nadeem Ahmed Sheikh

- Stefano Stella

- Kritika Subramanian

- Pieter Van Der Bijl junior

- Mauro Viganò

- Umar Zein

- Noam Zevit 